\section{HEAT TRANSFER ENHANCEMENT USING PASSIVE TECHNIQUE: REVIEW}

Adnan Rasheed Akeedya, Hajar Alias ${ }^{a *}$, Sami D. Salman ${ }^{b}$

aFaculty of Chemical Engineering, Universiti Teknologi Malaysia, 81310 UTM Johor Bahru, Johor, Malaysia

bAl-Khwarizmi College of Engineering, University of Baghdad, Baghdad, Iraa
Article history

Received

10 January 2020

Received in revised form

2 December 2020

Accepted

4 January 2021

Published online

23 February 2021

*Corresponding author

r-hajar@utm.my

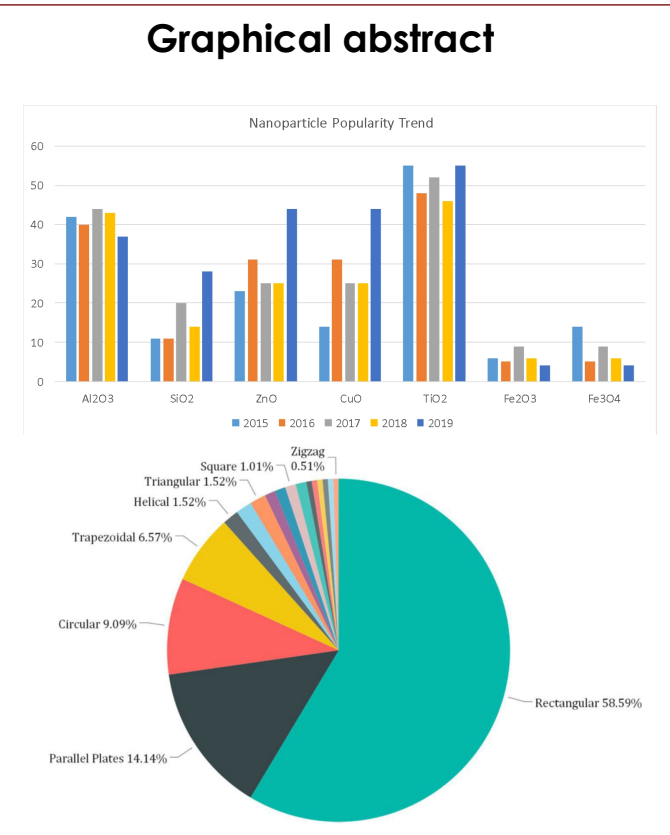

\begin{abstract}
Preserving and saving energy have never been more important, thus the requirement for more effective and efficient heat exchangers has never been more important. However, in order to pave the way for the proposal of a truly efficient technique, there is a need to understand the shortcomings and strengths of various aspects of heat transfer techniques. This review aims to systematically identify these characteristics two of the most popular passive heat transfer techniques: nanofluids and helically coiled tubes. The review indicated that nanoparticles improve thermal conductivity of base fluid and that the nanoparticle size, as well as the concentrations of the nanoparticles plays a major role in the effectiveness of the nanofluids. Regarding the helically coiled tubes, it was discovered that the use of a coiled tube produces secondary flows, which ultimately improves the heat transfer enhancement. The third part of the review focused on microchannels and microtubes. This is mainly due to the growing need and requirement of smaller and more compact thermal cooling systems. Thus, ultimately the result of the review indicates that a combination of all these three techniques can lead to a compact and minimized heat exchanger that uses the benefits obtained from both nanofluids and helically coiled tubes in order to improve the heat transfer rate of the thermal systems.
\end{abstract}

Keywords: Heat exhanger, heat transfer enhancement, nanoparticles, nanofluids, microtube, microchannel

(C) 2021 Penerbit UTM Press. All rights reserved

\subsection{INTRODUCTION}

In order to measure the thermal energy transferred between two matters, heat exchangers are employed. These matters could either be solid, gas or liquid, and are usually thermally connected with different temperatures [1,2]. Heat exchangers are often classified based on two main groups of techniques: active and passive [3]. When external power is employed, the technique is referred to as an active technique, otherwise, the technique is classified as passive. Examples of a passive technique are fluid additives such as nanofluids, tubes that are shaped in coil forms, surfaces that are extended, so on and so forth. Active technique examples, however, are more engaged and more expensive to use as well, such as electric fields and surface vibration [4]. There are also compound techniques that combine both passive and active techniques in order to produce effectiveness higher than if both techniques were to be used individually. 
Heat transfer enhancement techniques are considered to be an essential step to reduce the cost of energy and to be able to use the various energy sources to their maximum potential [5]. Previously, such techniques were employed in order to enhance the energy consumption of automobiles or other industries such as those that are involved in chemicals and processing. However, more recently, more cost - saving methods have been introduced in order to increase the heat exchanger performance. The three classifications of these techniques are used in order to achieve such a goal. Most of the attempts aimed at reducing both the cost and the size of such heat exchanger. Thus, the heat transfer coefficient is defined to have a direct correlation with the size and cost of the heat exchangers.

Ultimately, passive techniques are more desirable among the heat exchanger techniques [6]. This is due to the fact that passive techniques are far cheaper to implement than active techniques. In many instances, the cost of developing and implementing the equipment plays a major role in the usage of the technique. Thus, it is preferable to use passive techniques that rely not on external involvement, but rather a change in one of the main elements of the heat exchanger. One of the most popular and widely used techniques is the usage of nanoparticles and curved tubes in order to increase the performance rate of the heat exchanger via simple additions.

In this study, the aim is to systematically review two of the most popular passive techniques, which are the nanofluids and helical tubes. In order to achieve this, the methodology section elaborates on how this process was done, via the identification of research questions, and defining well and clearly established keywords and libraries in which the information and studies are selected from.

With the studies selected, the third section focuses on nanoparticles and nanofluids. It starts with a background on the field and then expands into the types and the various techniques that are used and implement them. The fourth section follows a similar procedure on the helical coil as a passive technique and uses the selected papers to elaborate how the helical coil enhances the heat transfer enhancement. In this section, the relevant studies are listed and summarized in a table as well as their unique contributions to the field. The fifth section focuses on microchannel and its impact on heat transfer enhancement. Each of these sections focuses on a brief background and overview, followed by a review and impact they have on the heat transfer rate. Finally, in conclusion, a summary of the reviewed paper is listed and their impacts analyzed as well as a prediction on future studies that can be spun from this review. Ultimately, there does not seem to be an indication that there are many techniques that use microtubes in helically coiled tubes. The benefits of such a combination are discussed in the conclusion of this review.

\subsection{REVIEW METHODOLOGY}

In order to provide a more structured flow for the review, a systemic approach is taken. Thus, research questions aim to provide that specific view point that is needed in order to collect the researches and conduct the review appropriately. However, these research questions are designed with the scope of passive techniques in mind. The goal is to understand how this group of techniques contributes to heat transfer effectiveness and what particular techniques ultimately provide the necessary benefit at the lowest cost and expense. The research questions are as the following:

(RQ1) What are nanoparticles and what is their effect on heat transfer exchange?

(RQ2) What is the effect of helical coil tubes in heat transfer effectiveness?

(RQ3) What are the benefits of microtubes when used in heat exchangers?

Thus, the review of this paper will be inducted base on the three main research questions mentioned above.

The source of the gathered researches is mainly papers that are indexed in Google Scholar. However, for the papers that are used for review, the most reputable libraries were prioritized. Libraries such as Science Direct, Elsevier, IEEE, ACM Digital Library and Springer are using as the main source of information.

The search criteria used for the general information and the introduction was using the Google Scholar search library and using the keyword "heat exchanger" and "heat transfer enhancement". These keywords are used independently in order to identify the relevant papers for the introduction. The date was of no concern when selecting these papers, as the goal is to get information on the background of the problem. Thus, the focus is not as strict as the other sections. The nanoparticle section uses two keywords of "nanofluid" and "nanoparticle", alongside keywords "heat transfer". This is necessary due to the fact that nanoparticles are used in many applications, and there is a need to narrow down the studies to only nanobased fluids and particles. The helical coil section also follows the same logic, by using the keyword "helically coiled" or "helical coil" alongside heat transfer, which narrows down the title to only heat transfer techniques. For the "microchannel" or "microtube" section, the keywords microchannel and microtube were used in conjunction with heat transfer and heat exchanger in order to select the papers that were related to this area and application of use.

For the review papers, only researches that were published after the years 2017 were used, with the exception of microtubes, were the year bracket $s$ between the years 2015 to 2019. These early papers were needed in order to provide a more chronological overview. 
Based on the search criteria outputs, 455 papers were extracted, however many were removed due to either redundancy of topic, or quality of the papers. In the case of such redundancies, libraries such as science direct, Elsevier, IEEE, ACM Digital Library, and Springer were prioritized. The goal was also to choose roughly 8 to 10 reviews that would demonstrate the review topic well enough. Thus, 52 papers have remained for review, of which, only 17 were systematically reviewed in the related sections, and the rest were used as background reference.

\subsection{NANOPARTICLES}

\subsection{Nanoparticle Background}

The definition of a nanoparticle is attributed to any particle or element that its size does not exceed 100 $\mathrm{nm}$ (nanometers), while it is also not smaller than 1 $\mathrm{nm}$. Due to their size, the nanoparticles exhibit particular properties that make them very desirable in many scientific fields. By using nanoparticles, some size-dependent properties are observed, some of these include but are not limited to quantum confinement, surface Plasmon resonance, superparamagnetic characteristics, and others. However, nanoparticles also are extremely useful for cases such as diffusion, which is mainly due to the high ratio of surface area to the volume. This allows nanoparticles to perform particular feats that could be useful in heat transfer related problems. Some of the metal-based nanoparticles combined with other fluids such as water, allow for the creation of nanofluids that have shown to improve the heat transfer enhancement [7]. The unique properties of these fluids and particles are elaborated further in this section.

Nanoparticles are classified as a passive heat transfer technique, since they are simply considered additives and are not external entities or energies that affect the system. Thus, it is a suitable and relatively cheaper alternative used for increasing the performance of heat transfer elements.

In heat transfer enhancement, there are metalbased nanoparticles that frequently being used. Some of these include particles from copper, silver, and gold, as well as some metal oxides, such as those of aluminum, iron, titanium and many more. There are several researches that incorporate nanofluids and nanoparticles in order to enhance the heat transfer rate [8]. Some of these, as well as the nanoparticle that they used in order to achieve this goal, are listed in Table 1.

Most of the present heat transfer enhancement seems to use mostly metal oxide, this is due to their unique structural characterizes, which makes them suitable for thermodynamic stability [8]. Although they also exhibit unique optical properties, for the purpose of heat transfer, such properties are irrelevant, and only their thermal and surface- related characteristics play a role in achieving the role of heat transfer enhancement.

Table 1 Common Nanoparticles

\begin{tabular}{|c|c|c|c|}
\hline Ref. & Author (year) & Nanoparticle & Name \\
\hline$[9,10]$ & $\begin{array}{c}\text { Manetti et al. (2017); } \\
\text { Pramuanjaroenkij et } \\
\text { al. (2018) }\end{array}$ & $\mathrm{Al} 2 \mathrm{O} 3$ & $\begin{array}{l}\text { Aluminum } \\
\text { Oxide }\end{array}$ \\
\hline [11] & Sundar et al. (2017) & $\mathrm{SiO} 2$ & $\begin{array}{l}\text { Silicon } \\
\text { Oxide }\end{array}$ \\
\hline$[11,12,13]$ & $\begin{array}{c}\text { Sundar et al. (2017); } \\
\text { Nadooshan (2017); } \\
\text { Askari et al. (2017) }\end{array}$ & $\mathrm{ZnO}$ & $\begin{array}{l}\text { Zinc } \\
\text { Oxide }\end{array}$ \\
\hline$[14,15]$ & $\begin{array}{l}\text { Sheikholeslami and } \\
\text { Sadoughi, (2018); } \\
\text { Karimzadehkhouei } \\
\text { et al. (2017) }\end{array}$ & $\mathrm{CuO}$ & $\begin{array}{c}\text { Copper } \\
\text { Oxide }\end{array}$ \\
\hline$[15,11]$ & $\begin{array}{l}\text { Karimzadehkhouei } \\
\text { et al.( 2017); Sundar } \\
\text { et al. (2017) }\end{array}$ & $\mathrm{TiO} 2$ & $\begin{array}{c}\text { Titanium } \\
\text { Oxide }\end{array}$ \\
\hline [11] & Sundar et al., (2017) & $\mathrm{Fe} 2 \mathrm{O} 3$ & $\begin{array}{l}\text { Iron (III) } \\
\text { Oxide }\end{array}$ \\
\hline$[4,16]$ & $\begin{array}{l}\text { Sheikholeslami and } \\
\text { Bhatti (2017); } \\
\text { Sheikholeslami and } \\
\text { Rokni (2018) } \\
\end{array}$ & $\mathrm{Fe} 3 \mathrm{O} 4$ & $\begin{array}{c}\text { Iron (II, III) } \\
\text { Oxide }\end{array}$ \\
\hline
\end{tabular}

\subsection{Review on Nanoparticles}

Nanofluids and nanoparticles have shown to enhance the heat transfer rate in various scenarios. However, several researches indicate that different concentrations yield different results in various scenarios when used nanoparticles. The effectiveness of these concentrations also depends on a variety of elements, such as the shape of the tube as well as the type of flow and even the main liquid that is used for the nanofluid mixture. Usually, the concentrations are between $0.01 \%$ minimum to $10 \%$ maximum with temperatures between 294 to 324 Kelvin.

Table 1 lists several studies that use a variety of nanoparticles, with some being tested under different volume fractions. However, most Heat Transfer Enhancement techniques, particularly those published in the past ten years, are always a combination of other techniques. Rarely there is research that focuses on the observation of a nanoparticle effect on its own, and in cases that it exists [17], the study is old.

The consensus among these old studies is that nanoparticles enhance the heat transfer rate in a considerable amount [18-21]. However, that amount depends on a variety of factors [22, 23]. Nanoparticles have often been observed to yield better results when combined with other passive or active techniques. Thus, in order to enhance the heat transfer rate further, most of the proposed techniques use a combination of two or more 
techniques, such as the usage of nanofluids with helically coiled tubes [24-27].

Earlier experiments use $\mathrm{ZnO}, \mathrm{MgO}$ and $\mathrm{CuO}$ nanofluids in conjunction with another passive technique by using a double pipe heat exchanger in a turbulent flow [28]. Under these conditions, CuO was observed to have a higher heat transfer enhancement rate than the other nanofluids. However, all of the listed nanofluids still outperformed water as the base fluid. Another experiment using a variety of heat exchanger coils was conducted using Aluminum Oxide $\left(\mathrm{Al}_{2} \mathrm{O}_{3}\right)$ [29]. This study was also conducted in a turbulent flow, with the results indicating that the velocity of the fluid has an effect on the effectiveness of the heat transfer technique. Although the main point of the technique revolves around the type of tube, it was discovered that the nanoparticle in use, was more effective than water.

Another researcher performs a numerical study of CFD method, followed by a single phase approach which is used in order to identify the effects of different concentration for the nanoparticles, in a turbulent flow [30]. The nanofluid was mainly comprised of $\mathrm{Fe}_{3} \mathrm{O}_{4}$ nanoparticles mixed with water. It shows that by using nanofluids, the results were significantly improved when compared to using only the base fluid. This improvement is further compounded with the usage of a circular cross section tube, as opposed to a straight or triangular channel.

A research experiment on the properties and characteristics of a copper-based heat transfer oil nanofluid was conducted under laminar flow [31]. The results indicate that under certain Richardson numbers, the Nusselt number will increase as the weight concentration of the nanoparticles is also increased. The use of iron (II, III) oxide $\left(\mathrm{Fe}_{3} \mathrm{O}_{4}\right)$ nanoparticles were experimented with different concentrations under turbulent flows [32]. The nanoparticle was mixed with water at four different concentrations of $0.06 \%, 0.03 \%, 0.01 \%$ and $0.005 \%$. Based on the discovered results, new correlations were identified between Nusselt number, friction factor and various nanofluid concentrations.

\subsection{Nanoparticle Research Trends}

For a better understanding of the direction of the Nanofluids, a trend analysis is used that evaluates the recent trends and popularity metrics of the nanofluids listed in Table 1. Although there are more nanofluids used, these are the most popular ones. Figure 1 illustrates a bar chart that lists all the existing literature from top libraries such as science direct and Elsevier. The search criteria were used to select papers that focused on nanofluid as an enhancement tool for heat transfer. There are several papers that use nanofluid as other forms and solutions, however, the purpose of this research is to identify the related heat transfer enhancement researches. Thus, Figure 1 bar chart illustrates how each of these nanofluids was used between the years 2015 and 2019. The focus of these researches was primarily on the selected nanoparticle. For instance, if a paper or research is doing a mere review, it is skipped. However, if the paper is based on simulation or an experiment that pertains to the nanoparticle only, then it is selected.

Based on the observed trends, some of the nanofluids seem to be more used than the others. Interestingly enough, the trend indicates that some nanoparticles have kept their popularity constant throughout the last five years. This is particularly the case for nanoparticles such as aluminum oxide and titanium oxide. Both of these particles seem to be used on average around 40 for aluminum oxide and 50 research studies on titanium oxide. They have been relatively in the same trend and there doesn't seem to be a pattern that would indicate an increase. There has been little to no interest in the variety of Iron Oxide (II) and (III). Consequently, there is an observed increase in popularity for zinc oxide, copper oxide, and silicon oxide. All of these nanoparticles seem to have had a large increase in the last year alone which could indicate their usefulness. Figure 2 illustrates a pie chart of the total number of researchers in the last five years. This shows that nanoparticles such as aluminum oxide and titanium oxide are among the most used and popular ones. This could relate both to their relatively low expense, and their effectiveness of results. 


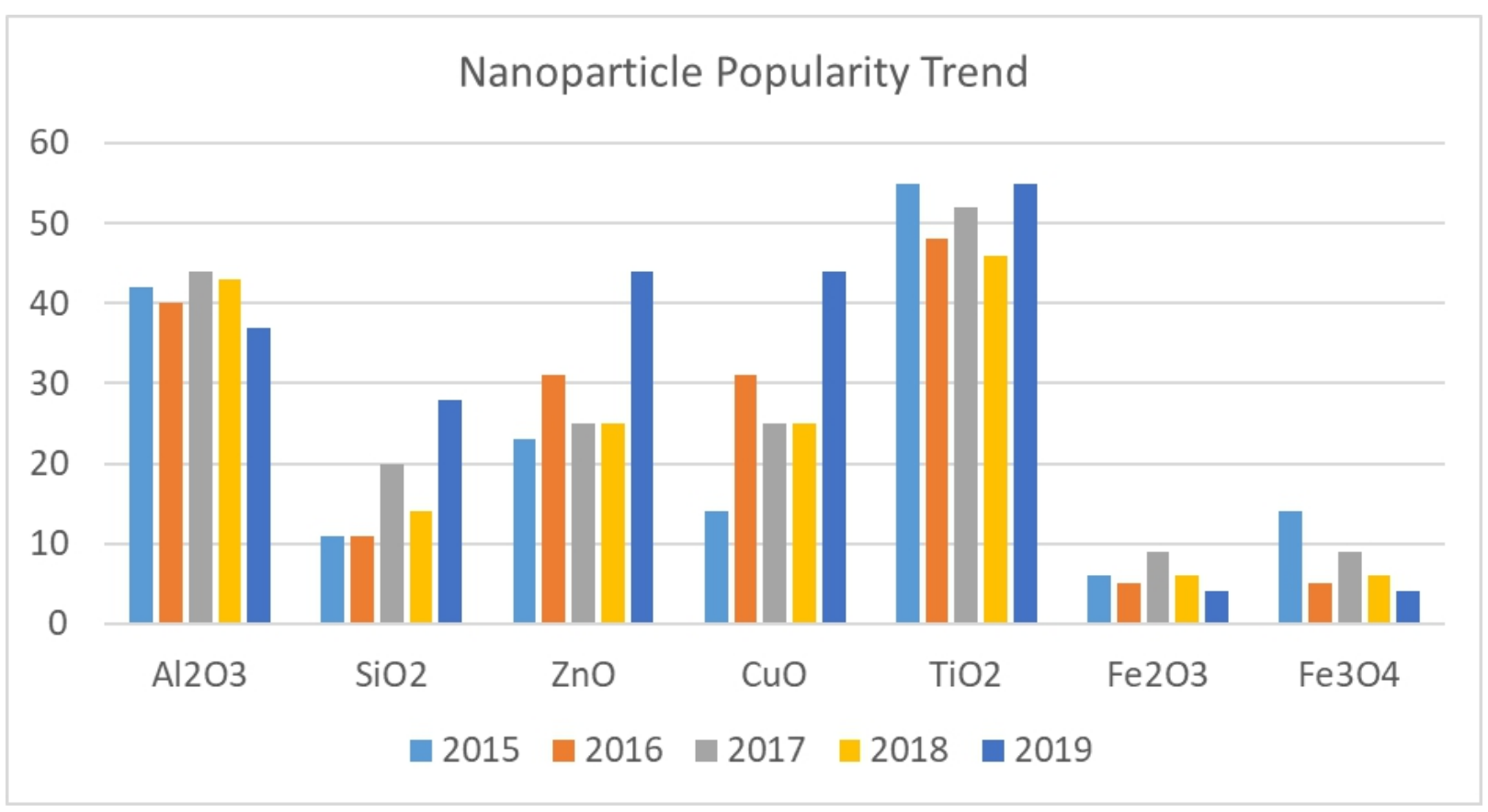

Figure 1 Nanoparticle Research Popularity in the last five years

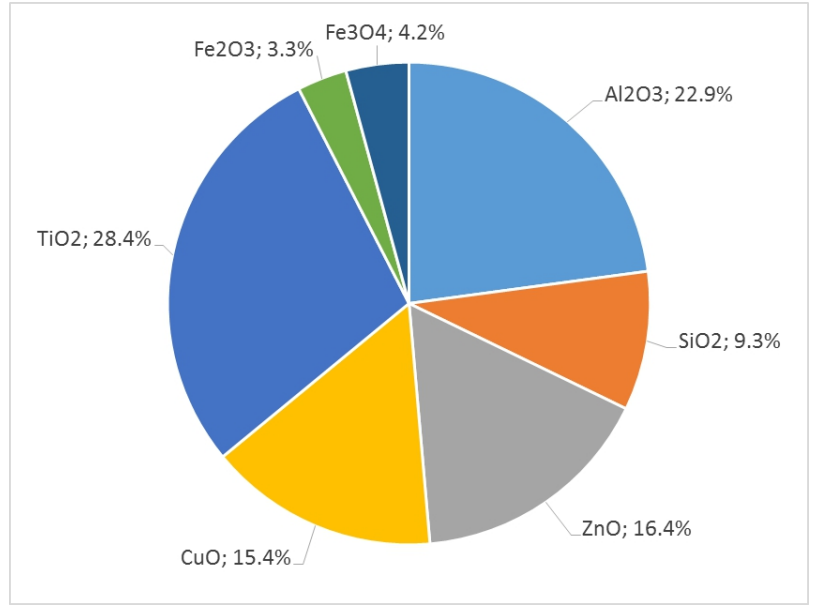

Figure 2 Nanoparticle Popularity Percentage

\subsection{Discussion}

Based on the reviewed researches, the observations from both numerical and experimental findings, indicate that the inclusion of nanoparticles improve the thermal conductivity and heat transfer rate without actually changing the physical or chemical properties of the base liquid. However, it is not possible to determine which nanoparticle is best, as each behaves differently in different situations. For instance, in a listed example copper oxide outperformed the other nanoparticles, however, this may not be the case in all instances. The indication is that there are several factors that affect the performance of the nanoparticles. Some are listed here.

The Reynolds Number, which dictates whether the flow is laminar or turbulent has been an indicator of nanoparticle performance. While in some instances it has been seen to perform better in turbulent flows, there are examples of the particles performing similarly in the laminar flow under other factors. The viscosity of the fluid is another contributing factor, as well as the concentration of the nanoparticle in the nanofluid. The tube shape also seems to play a role in how a particular nanoparticle behaves.

Ultimately, there is an indication that nanoparticles are superior when compared to base fluids such as water and even in some cases, gas. Thus, the use of such form of fluids is highly recommended for any technique that intends on improving the performance of the heat exchange unit.

\subsection{HELICAL COIL ENHANCEMENT TECHNIQUES}

\subsection{Helical Coil Background}

The need for a more compact and efficient design for heat exchangers is an ever growing problem which is currently on the rise among the researchers in the field. This is an important issue, as the size becomes smaller and more compact, the need for a consistently higher performance becomes imperative. As elaborated earlier, there is a wide range of factors that affect the performance of the 
heat transfer rate. Some of these are related to the fluid that is used, and some relate to the shape of the tube. This of course, is only considering the passive techniques group, as the active techniques are out of scope.

Thus, this requirement for a more compact design, leads to the introduction of helically coiled tubes which have been proven to enhance the heat transfer and apply itself in a variety of industries, whilst staying compact. The formation of the secondary flow, which is an intended feature of the design, the fluid mixing process is enhanced and thus, when compared to the straight tube heat exchanger, it yields better results.

\subsection{Helical Coil Techniques Review}

In order to gain a better understanding of these passive techniques, the following experiments are listed and elaborated in detail on the effectiveness and efficiency of the helically coiled tubes. These techniques, which use helical coiled tubes, however, each have a certain change and different in some parameters. All techniques are listed and summarized on in Table 2.

Table 2 Helical Coil Heat Exchanger Performance

\begin{tabular}{|c|c|c|c|c|c|c|}
\hline Ref. & Author (year) & Study & Tube Type & Fluid & Flow & Result Indication \\
\hline [25] & $\begin{array}{l}\text { Fule et al. } \\
(2017)\end{array}$ & Exp. & Helical Coil & CuO/Water & Laminar & $\begin{array}{l}\text { Improved HTR due to increased } \\
\text { flow rate of nanofluid }\end{array}$ \\
\hline [26] & $\begin{array}{l}\text { Palanisamy } \\
\text { and Kumar, } \\
\text { (2017) }\end{array}$ & Exp. & $\begin{array}{l}\text { Helical Coil - Cone } \\
\text { Shape }\end{array}$ & $\begin{array}{l}\text { MWCNT } \\
\text { /Water }\end{array}$ & Laminar & $\begin{array}{l}\text { The cone shape impacts the } \\
\text { thermal conductivity and } \\
\text { enhances heat transfer }\end{array}$ \\
\hline [33] & $\begin{array}{l}\text { Etghani and } \\
\text { Baboli, ( 2017) }\end{array}$ & Num. & Helical Tube & Water & Laminar & $\begin{array}{l}\text { Pitch coil, tube diameter fluid } \\
\text { temperature affect HTC }\end{array}$ \\
\hline [34] & $\begin{array}{l}\text { Wen et al., } \\
\text { (2017) }\end{array}$ & Exp. & $\begin{array}{l}\text { Vertical Helical } \\
\text { Tube }\end{array}$ & Water & Turbulent & $\begin{array}{l}\text { HTC is larger on the outside } \\
\text { than inside }\end{array}$ \\
\hline [35] & $\begin{array}{l}\text { (Panahi and } \\
\text { Zamzamian, } \\
2017 \text { ) }\end{array}$ & Exp. & $\begin{array}{l}\text { Shell and Coiled } \\
\text { Tube }\end{array}$ & $\begin{array}{l}\text { Water } \\
\text { Air }\end{array}$ & Turbulent & $\begin{array}{l}\text { HTC improved substantially due } \\
\text { to tube shape }\end{array}$ \\
\hline [36] & $\begin{array}{l}\text { Zhang et al., } \\
(2017)\end{array}$ & Num. & $\begin{array}{l}\text { Helically coil tube } \\
\text { with spherical } \\
\text { corrugation }\end{array}$ & Water & Turbulent & $\begin{array}{l}\text { HT Process improved } \\
\text { significantly due to corrugation } \\
\text { structure }\end{array}$ \\
\hline [37] & $\begin{array}{l}\text { Fu et al., } \\
(2017)\end{array}$ & Exp. & $\begin{array}{l}\text { Helical Tube with } \\
\text { different diameters } \\
\text { but fixed pitch }\end{array}$ & Water & Turbulent & $\begin{array}{l}\text { The outer HTC reduces } \\
\text { gradually but is less than inner } \\
\text { side due to coking disposition }\end{array}$ \\
\hline [38] & $\begin{array}{l}\text { Palve and } \\
\text { Kale,( 2015) }\end{array}$ & Num. & $\begin{array}{l}\text { Helical Coil Copper } \\
\text { Tube }\end{array}$ & Water & Turbulent & $\begin{array}{l}\text { The geometry of the helical coil } \\
\text { heat exchanger increases } \\
\text { pressure drop }\end{array}$ \\
\hline
\end{tabular}

The first study revolves around the effects of tube diameter and mass flow rate on the effectiveness of the heat transfer [38]. The experiment was performed using a helical coil heat exchanger, and the simulation was performed using a CFD methodology. The application used to perform the simulation is ANSYS FLUENT version 15.0. The main configuration for the simulation is to choose the material for the tube as copper, and the main fluid chosen for the system is water. The experiment has been conducted by varying pipe diameter sizes, with a constant pitch. The temperature is also set at 343K. Based on the observed results, in a helical coil heat exchanger, the geometry has an effect on both temperature and pressure. Depending on the tube diameter, if it is reducing in size then there is an increase in temperature drop, while the pressure drop goes on increasing.

In the second study, a group of researchers investigated a numerical model of shell and helical tube heat exchanger to assess heat transfer coefficient and exergy loss [33]. Based on the reported results, there is an indication that the diameter of the tube and cold temperature of the flow are very important parameters that have a major impact on heat transfer and energy loss (respectively). The heat transfer coefficient is observed to be reduced as the pitch of the coil is increased, as well as the hot flow rate, which results in increased energy loss. Based on the recorded results, an optimum level is discovered for the heat transfer coefficients. These values are: pitch $13 \mathrm{~mm}$, tube diameter $12 \mathrm{~mm}$, cold and hot flow rate 4 liter per minute. Furthermore, the optimum level for exergy loss is: pitch $13 \mathrm{~mm}$, tube diameter $12 \mathrm{~mm}$, cold and hot flow rate 1 liter/min.

In the third study, a researcher conducted an experimental investigation on helical tubes for aviation kerosene RP-3 [37]. For the experiment, four different types of helical tubes are chosen, however 
the pitch is a fixed value, the diameters have a 1.86 $\mathrm{mm}$ inner diameter and $2.2 \mathrm{~mm}$ outer diameter. The system pressure, inlet temperature, mass flow rate was fixed at $5 \mathrm{MPa}, 400 \mathrm{~K}$ and $1178 \mathrm{~kg} / \mathrm{m}^{2} \mathrm{~s}$, respectively. Based on the observed results, the helical centrifugal force could inhibit the thermal oxidation coking and slow down the growth rate of press drops within the maximum value of $47.6 \%$. For the same cross section, the outer heat transfer coefficient (HTC) decreases gradually and eventually is less than the inner side due to coking deposition.

The fourth study conducted a simulation on helically coiled tube's heat transfer and pressure drop, however the difference with other researches is that the tube is augmented with a spherical corrugation [36]. Based on the results simulated, the flow that is induced due to the centrifugal force has a high impact on the heat transfer rate enhancement. The heat transfer process is increased due to the corrugation structure causing an eddy, which increases the turbulence intensity, and ultimately enhances the heat transfer process. With the increase of the corrugation height $(H)$, the augmentation on heat transfer performance is about 1.05-1.7 times as compared to the smooth helically coiled tube, while friction factor sharply increases 1.01-1.24 times. With the increase of the corrugation pitch $(P)$, the enhancement on heat transfer is in the range of 1.37-1.66 times and the friction factor increases 1.18-1.28 times compared to the smooth helically coiled tube. The overall heat transfer performance of helically coiled tube with corrugation is better than that of smooth helical tube under the same condition. The value of the performance evaluation criterion (PEC) could be up to 1.56 .

The fifth studied is experimental research on the shell and coiled tube heat exchanger [35]. This particular experiment uses a helical wire as a turbulator inside the helical coil. The experiment was conducted using water once and air the second in the coiled tube. Both of these mods were experimented with and without the turbulator, meaning that the wire was removed once and inserted again for testing. The water temperature was set as hot for all the modes in which it was tested. Based on the results, the heat transfer coefficient was significantly improved with the use of the turbulator.

The final study summarized here conducted a research on convective heat transfer characteristics of aviation kerosene RP-3 at supercritical pressure (P $=5 \mathrm{MPa}$ ) in vertical helical tube [34]. Based on the reported results, the inside temperature is higher than the outside due to the force caused by the secondary flow centrifuge. Hence, the heat transfer coefficient is larger about $31.5 \%$ when compared the inside and the outside centrifuges. Furthermore, heat transfer enhancement leading by centrifugal secondary flow is the key factor when the Richardson ratio is larger than 10. At last, two correlations of Nusselt number were developed to predict heat transfer of aviation kerosene RP-3 in helical tube based on experimental data at supercritical pressure.

\subsection{Discussion}

Based on the observed results from the review section as well as the summarized information listed in Table 2, it can be concluded from this section that the helically coiled tubes improve the performance of the heat exchange rate. An important note that can be easily observed in Table 2 is that most of the techniques use either water, or air, and only two of the listed techniques use alternate fluids such as nanofluids. This is important, as the goal of this section is to make sure that helically coiled tubes improve the performance regardless of the fluid used, and in fact, if there were to be a fluid used as an additive to the technique, the performance would be even higher.

Overall, the techniques indicated that compared to the base straight shape, the helical coil is both more compact and far more effective in practice. The effectiveness factor comes from the secondary flow that is caused due to the curvature of the pipe, which as elaborated earlier, causes the fluid to mix in a more effective way.

\subsection{MICROCHANNEL HEAT EXCHANGERS}

\subsection{Microchannel Introduction}

Heat transfer enhancement as a field has grown over the years and it has been included in many applications and fields. This has prompted researchers to push development for a more compact effective means of fluid flow, thus microchannels and microtubes have been the most recent system that aims to provide the aforementioned benefits [24]. Thus, understanding and using these phenomena is imperative for creating such systems.

Microtubes and microchannels being used as cooling devices are the next logical step in engineering, as the demand for smaller and more compact design leads the way to a more effective and efficient heat transfer rate per unit volume. This is particularly useful for microchip cooling, which is extremely popular in computer engineering and a must have-piece of technology since the space is relatively constrained. Other microchannel and microtube applications include, but are not limited to: automobile, transportation and biomedical industries, as well as broader usages in refrigeration systems [39], micro-reactors [40], transformers [41], solar collectors [42] and many more [43].

Microtubes are ultimately reliant on an efficient thermal design that would optimally take in the conditions of a certain scenario and ultimately set the diameter based on those requirements. Thus, this section focuses on reviewing such an important 
piece of technology and how it has been used over the past few years as a research field.

\subsection{Microchannel Review}

Initially, the microchannel technology as a heat transfer tool was proposed for the purpose of microchip cooling with the use of water as fluid [44]. Thus, the demand for a thermal management system used for electronic systems increased, as the sizable and compact cooling systems allowed for more efficient and compact designs to be implemented. Over the past decades, some of the popular liquids used with the microchannel heat transfer units are water, air, Ethylene Glycol, and other forms of fluids.

The classification of microchannels usually falls into four categories of micro, meso, compact and conventional. This classification is performed based on the diameter of the channels. This study aims to review the recent uses and discoveries of the microchannels in heat exchangers and how they have benefited the systems not only in terms of size, but also in terms of performance and effectiveness. This ultimately leads to the identification of strengths and weaknesses of the microchannels and how they can be applied better into other aspects of heat transfer. One of the important aspects of the microchannels is also the unique geometry that they use in order to transport fluids, which in some cases can create particular effects depending on the type of fluid and flow that is used.

Table 3 lists some of the recent studies that have been done in heat exchanger techniques using a variety of tube shapes and nanoparticles. These are tested in various flows, defined by their Reynolds Number. Some of the techniques are purely numerical, while some are experimental. There are those that also have both experimental and numerical.

Table 3 Review of the Recent Literature in Microchannel Heat Exchanger Techniques

\begin{tabular}{|c|c|c|c|c|c|c|}
\hline Ref. & Author (year) & Study & Tube Type & Nanofluid & Flow & Result Indication \\
\hline$[45]$ & $\begin{array}{l}\text { Sivakumar et al., } \\
\text { (2014) }\end{array}$ & $\begin{array}{l}\text { Exp. } \\
\text { Num. }\end{array}$ & $\begin{array}{l}\text { Serpentine Shaped } \\
\text { Microchannel }\end{array}$ & CUO/EG & Laminar & $\begin{array}{l}\text { Improvement observed } \\
\text { when compared to } \\
\text { base fluid only. }\end{array}$ \\
\hline$[46]$ & $\begin{array}{l}\text { Ebrahimi et al., } \\
(2016)\end{array}$ & Num. & $\begin{array}{l}\text { Rectangular } \\
\text { Microchannel }\end{array}$ & $\begin{array}{l}\left(\mathrm{Al}_{2} \mathrm{O}_{3}, \mathrm{CuO}\right) \\
\text { /Water }\end{array}$ & $\begin{array}{l}\text { Laminar } \\
\text { Turbulent }\end{array}$ & $\begin{array}{l}\text { Improvement for both } \\
\text { nanofluids observed. }\end{array}$ \\
\hline [47] & $\begin{array}{l}\text { Akbari et al.. } \\
(2016)\end{array}$ & Num. & $\begin{array}{l}\text { Microtube } 90^{\circ} \\
\text { Curvature Angle }\end{array}$ & CuO/Water & Laminar & $\begin{array}{l}\text { The shape's buoyancy } \\
\text { and centrifugal forces } \\
\text { improve HT }\end{array}$ \\
\hline$[48]$ & $\begin{array}{l}\text { Behnampour et } \\
\text { al., (2017) }\end{array}$ & Num. & $\begin{array}{l}\text { Triangular, } \\
\text { Rectangular, } \\
\text { Trapezoidal Ribs }\end{array}$ & Ag/Water & Laminar & $\begin{array}{l}\text { Trapezoidal shape more } \\
\text { effective for higher } \\
\text { Reynold Numbers. }\end{array}$ \\
\hline [49] & $\begin{array}{l}\text { Sajadifar et al., } \\
(2017)\end{array}$ & Num. & Microtube & $\mathrm{Al}_{2} \mathrm{O}_{3} / \mathrm{CMC}$ & Laminar & $\begin{array}{l}\text { More volume fraction } \\
\text { and slip coefficient } \\
\text { relate to higher NN }\end{array}$ \\
\hline [15] & $\begin{array}{l}\text { Karimzadehkhovei } \\
\text { et al.,( 2017) }\end{array}$ & Exp. & $\begin{array}{l}\text { Horizontal } \\
\text { Microtube }\end{array}$ & $\mathrm{Y}-\mathrm{Al}_{2} \mathrm{O}_{3} /$ Water & Laminar & $\begin{array}{l}\text { Nanoparticle } \\
\text { concentration an } \\
\text { important factor on HT }\end{array}$ \\
\hline [20] & $\begin{array}{l}\text { Akbari et al., } \\
\text { ( 2017) }\end{array}$ & Num. & $\begin{array}{l}\text { Rectangular 2D } \\
\text { Microchannel }\end{array}$ & $\mathrm{Al}_{2} \mathrm{O}_{3} / \mathrm{CMC}$ & Laminar & $\begin{array}{l}\text { Reduction in the } \\
\text { diameter of the } \\
\text { nanoparticles improves } \\
\text { HT }\end{array}$ \\
\hline$[50]$ & $\begin{array}{l}\text { Karimzadehkhovei } \\
\text { et al., (2018) }\end{array}$ & $\begin{array}{l}\text { Exp. } \\
\text { Num. }\end{array}$ & Microtube & $\mathrm{Y}-\mathrm{Al}_{2} \mathrm{O}_{3} /$ Water & Laminar & $\begin{array}{l}\text { Nanoparticles } \\
\text { performed better at } \\
\text { higher Reynold Numbers }\end{array}$ \\
\hline$[51]$ & $\begin{array}{l}\text { Rahimi Gheynani } \\
\text { et al., (2018) }\end{array}$ & Exp. & 3D Microtube & $\mathrm{CUO} / \mathrm{CMC}$ & Turbulent & $\begin{array}{l}\text { Reduction in the } \\
\text { diameter of the } \\
\text { nanoparticles improves } \\
\text { HT }\end{array}$ \\
\hline
\end{tabular}

Although not entirely unexpected, one of the most popular methods used to improve the performance of microchannels is the use of nanofluids and nanoparticles. Thus, it comes to no surprise when all of the listed researches used nanofluids in some form and capacity. The first research aimed on investigating the use of copper oxide mixed with ethylene glycol $\left(\mathrm{CH}_{2} \mathrm{OH}\right) 2$ in a serpentine shaped microchannel using laminar flow. The concentration of the nanoparticle is only $0.3 \%$, however, the results indicated that the fluid mixed with the shape of the microchannel improve on the effectiveness when compared to a base form without the nanoparticles and the shape in question [45]. 
The second research also used nanoparticles, however this time other than copper oxide, there was also aluminum oxide added, and the base fluid used was water, rather than ethylene glycol. The shape of the microtube is also different, with this one having rectangular-shaped microchannel [46]. The concentrations used were much more varied than the previous research, with them varying between $0.5 \%$ to $3.0 \%$ of nanoparticles with varying sizes dispersed in pure water. The results were indicating a positive increase for both nanofluids, in terms of heat transfer enhancement. In this unique experiment, both laminar and turbulent flows were tested. However, the experiment was ultimately only numerical in nature.

In the third study, copper oxide and water are used again, however, the microchannel in question has a 90 degree curvature angle [47]. The flow used was laminar, with nanoparticle concentrations varying from $0 \%$ to $6 \%$. The numerical study was expensive and experimented with a variety of characteristics, which ultimately led to the verification of both nanoparticles and microchannels having a positive effect on the heat transfer rate of the system.

The fourth numerical study used a combination of silver nanoparticles in water and microtubes that are triangular, rectangular and trapezoidal in shape [48]. The nanoparticle concentrations tested were $0 \%$ (meaning testing only water), $2 \%$ and $4 \%$. Based on the reported results, the thermal performance of the shapes was least successful for rectangular ribs, and most successful for trapezoidal ribs. This is particularly true when the Reynolds numbers were higher in value.

The fifth numerical experiment uses a microtube with nanoparticles mixed in carboxymethyl cellulose (CMC). This time the nanoparticles are aluminum oxide [49]. The focus of this experiment was mainly on the tube itself, with a length of 5 millimeters and a diameter of 0.2 millimeters. The flow tested in this experiment was mainly laminar with the results indicating that more volume fraction and slip coefficient correspond to higher Nusselt number, which is more true when the Reynolds number is higher.

The sixth experiment is not numerical, but rather a form of the review itself on the use of a horizontal microtube mixed with the use of nanofluids of gamma-alumina nanoparticles mixed with water. The diameters of the nanoparticle included were 20 nanometers. This experiment observed that higher mass fractions on nanofluids deteriorated the heat transfer rate [15]. Observations of dynamic light scattering measurements for low and high mass fractions before and after the experiments revealed that agglomeration of nanoparticles are an important parameter in the deterioration of heat transfer at higher concentrations.

The seventh experiment is numerical in nature, and aimed on investigating the behavior of nanofluids in a rectangular 2D microchannel [20].The nanoparticle used is a mixeture of aluminum oxide mixed with carboxyl methyl cellulose (CMC). This microchannel heat exchanger was tested under laminar flow with Reynolds number between 10 to 1000. Based on the results, the increase in volume fraction and a reduction in the diameter of the nanoparticles ultimately lead to a higher heat transfer rate.

More recently, the eight researches performed a study both experimental and numerical that used microtubes and nanoparticles [50]. However, this particular experiment used deionized water and gamma-alumina as their nanofluid with a mass fraction of $0.1 \%$ and varying diameter sizes between 1 and 100 nanometers. Both experimental and numerical results were in agreement, and concluded that the inlet temperature effect was more pronounced for the thermally developing region. The performance enhancement with nanoparticles was obtained at rather higher Reynolds Numbers and near the inlet of the microtube.

The final study used 3D microtubes with conjunction of nanofluids composed of copper oxide and CMC [51]. The results of this study were observed under turbulent flows, which differ from the previous studies. The focus of this study relied on the diameter of the nanoparticles used, which were 25 nanometers and 50 nanomaterials with concentration increasing from $0.5 \%$ to $1 \%$. The results ultimately indicated that a lower diameter improved the performance more notably than the higher diameter compared.

\subsection{Discussion}

Ultimately, microchannels and microtubes are shown to be extremely useful for a variety of thermal systems, particularly those in computer engineering. The reviews indicated that the use of microchannels mixed with other types of techniques, such as changing the shape and direction of the pipe, as well as using a variety of different nanoparticles and nanofluids yield a positive result both numerically and experimentally. The ultimate takeaway is that the diameter of the nanoparticles used played a major role, with smaller sizes performing better than those with bigger size particles. This is particularly a good effect for microchannels which are already a smaller size, and benefit greatly from effects that provide such benefit. Thus, this findings suggest that the use of microchannels an interesting and important point.

Figure 3 illustreates the microchannel geometry share from a recently published review. Based on the results of the review, the pie chart illustrated in Figure 3 shows that helically coiled microchannel are used only in $1.52 \%$ of the total researches. Making them very rare as of 2018. Further research in 2019 has also shown that only a few researches have been published in the area. This further reinforces the idea that more research is needed in the helically coiled microchannels. 


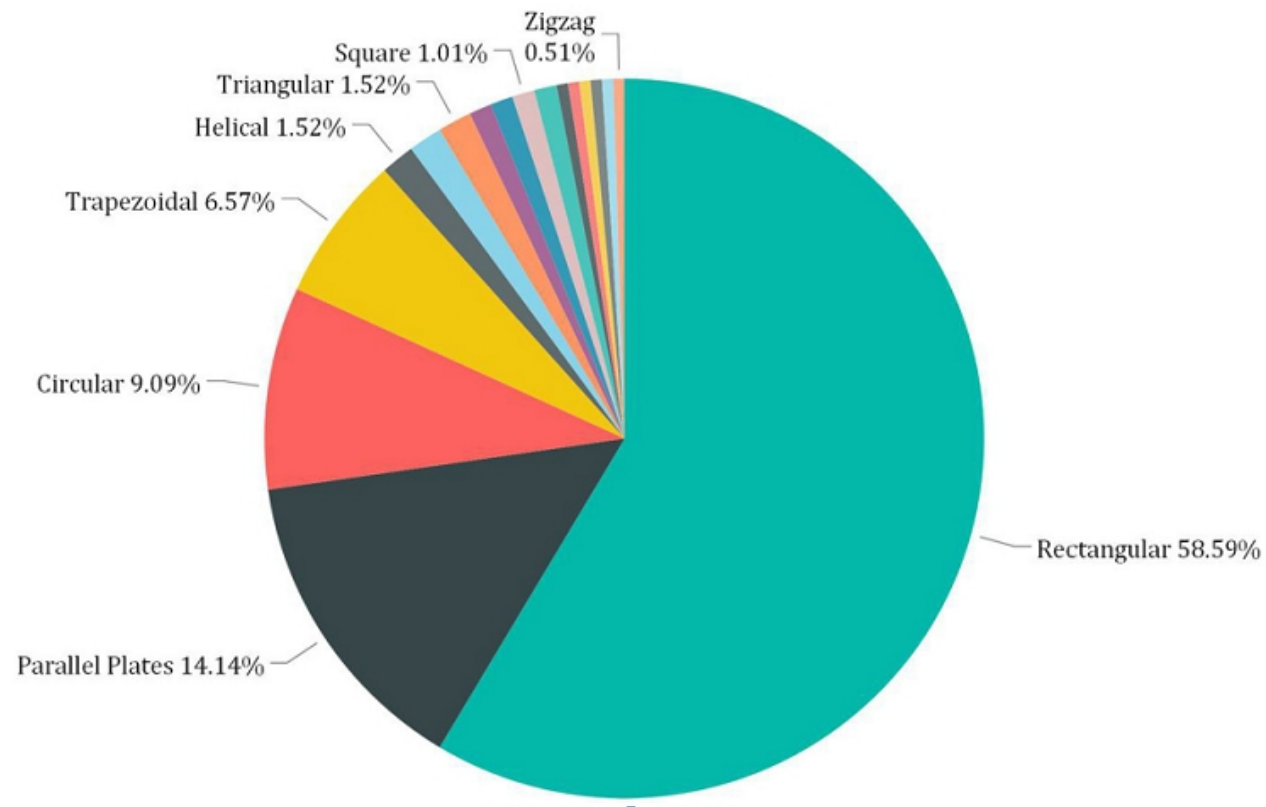

Figure 3 Microchannel Geometry Popularity [52]

\subsection{CONCLUSION}

This study performed a review on three different, yet related topics that affected heat transfer. Various numerical and experimental researches were studied under different passive heat transfer techniques that aimed on improving the performance of the heat exchangers in one way or another.

The first group of techniques measured was the nanofluids and nanoparticles, which proved to be an extremely useful technique for improving the performance of the heat exchangers. The second technique was the use of helically coiled tubes, which also had an effect in improving the heat transfer rates. The final category was the use of microchannels which are an important piece of technology that affects many new and popular areas of research.

The results of this review lead to several discoveries and understandings of the passive techniques for heat transfer rates which are:

1.The first focus on nanoparticles identified them as a suitable technique that is often used in mixture of other active and passive techniques in order to improve the performance of the heat exchangers. This is due to their cheap and inexpensive implementation and their unique characteristics. Ultimately, the results indicated that smaller nanoparticles have a greater effect on the heat transfer rates and their concentration also plays a major role in their usage. However, these values greatly depend on other aspects of the technique, such as the Reynolds Number and the property characteristics unique to the case under test.
2. Helically coiled tube heat exchangers were reviewed without the use of the nanoparticles in order to gain a better understanding of how they perform. The results were positive and indicated that this type of technique can improve the heat transfer rate of thermal systems on their own.

3. The previous studied indicated that the use of a coiled tube produces secondary flows which promote higher heat transfer coefficients in single-phase flows as well as in most regions of boiling. The presence of nanofluid improves the effective thermal conductivity, leading to a significant increase in convection heat transfer coefficient.

4. Microchannels and microtubes are excellent forms of evolution for thermal systems that allows them to be more compact and effective in design. Combining other passive techniques into the microchannel and microtube design as well as the usage of coiled shapes such as those reviewed in helically coiled heat exchangers can lead to a new design that is both compact and effective in nature.

Ultimately, this review identifies the attractive characteristics of nanofluid in helical tube heat exchangers, as well as the benefits of microtubes and microchannels have encouraged the current research to propose a combination of all three of this elements into a single method which encompasses all of the benefits as well as extra ones that is carried on depending on the case that is being tested. This can provide even more improvement when compared to the individual use of micro coil with nanofluid or microtubes and microchannels (MT/MC) 
with nanofluid flow. Also, as alluded to earlier, the combination of nanofluid with the new configuration for the micro coil tube could be result in a higher degree of performance than the other forms of conventional liquids such as water, oil or ethylene glycol.

\section{Acknowledgement}

The authors would like to express gratitude to the Malaysia Ministry of Education (MOE) and Universiti Teknologi Malaysia for Research University Grant ( $Q$ JI30000.2546.19H46).

\section{References}

[1] Şimşek, E., Coskun, S., Okutucu-Özyurt, T. \& Unalan, H. E. 2018. Heat Transfer Enhancement by Silver Nanowire Suspensions in Microchannel Heat Sinks. International Journal of Thermal Sciences. 123: 1-13.

[2] Sheikholeslami, M., Ashorynejad, H. \& Rana, P. 2016. Lattice Boltzmann Simulation of Nanofluid Heat Transfer Enhancement and Entropy Generation. Journal of Molecular Liquids. 214: 86-95.

[3] Sheikholeslami, M., Gorji-Bandpy, M. \& Ganji, D. D. 2015. Review of Heat Transfer Enhancement Methods: Focus on Passive Methods Using Swirl Flow Devices. Renewable and Sustainable Energy Reviews. 49: 444-469.

[4] Sheikholeslami, M. \& Bhatti, M. 2017. Active Method for Nanofluid Heat Transfer Enhancement by Means of EHD. International Journal of Heat and Mass Transfer. 109: 115122.

[5] Alam, T. \& Kim, M.-H. 2018. A Comprehensive Review on Single Phase Heat Transfer Enhancement Techniques in Heat Exchanger Applications. Renewable and Sustainable Energy Reviews. 81: 813-839.

[6] Sidik, N. A. C., Muhamad, M. N. A. W., Japar, W. M. A. A. \& Rasid, Z. A. 2017. An Overview of Passive Techniques for Heat Transfer Augmentation in Microchannel Heat Sink. International Communications in Heat and Mass Transfer. 88: 74-83.

[7] Rashidi, S., Eskandarian, M., Mahian, O. \& Poncet, S. 2018. Combination of Nanofluid and Inserts for Heat Transfer Enhancement. Journal of Thermal Analysis and Calorimetry. 1-24.

[8] Bianco, V., Vafai, K., Manca, O. \& Nardini, S. 2015. Heat Transfer Enhancement with Nanofluids. CRC Press.

[9] Manetti, L. L., Stephen, M. T., Beck, P. A. \& Cardoso, E. M. 2017. Evaluation of the Heat Transfer Enhancement during Pool Boiling Using Low Concentrations of $\mathrm{Al}_{2} \mathrm{O}_{3}$-water based Nanofluid. Experimental Thermal and Fluid Science. 87: 191-200

[10] Pramuanjaroenkij, A., Tongkratoke, A. \& Kakaç, S. 2018. Numerical Study of Mixing Thermal Conductivity Models for Nanofluid Heat Transfer Enhancement. Journal of Engineering Physics and Thermophysics. 91: 104-114.

[11] Sundar, L. S., Sharma, K., Singh, M. K. \& Sousa, A. 2017. Hybrid Nanofluids Preparation, Thermal Properties, Heat Transfer and Friction Factor-A Review. Renewable and Sustainable Energy Reviews. 68: 185-198.

[12] Nadooshan, A. A. 2017. An Experimental Correlation Approach for Predicting Thermal Conductivity of WaterEG based Nanofluids of Zinc Oxide. Physica E: Lowdimensional Systems and Nanostructures. 87: 15-19.

[13] Askari, S., Koolivand, H., Pourkhalil, M., Lotfi, R. \& Rashidi, A. 2017. Investigation of Fe3O4/Graphene Nanohybrid Heat Transfer Properties: Experimental Approach. International Communications in Heat and Mass Transfer. 87: 30-39.
[14] Sheikholeslami, M. \& Sadoughi, M. 2018. Simulation of CuO-water Nanofluid Heat Transfer Enhancement in Presence of Melting Surface. International Journal of Heat and Mass Transfer. 116: 909-919.

[15] Karimzadehkhovei, M., Sezen, M., Şendur, K., Mengüç, M.P. and Koşar, A. 2017. Subcooled Flow Boiling Heat Transfer of $\mathrm{Y}$-Al2O3/water Nanofluids in Horizontal Microtubes and the Effect of Surface Characteristics and Nanoparticle Deposition. Applied Thermal Engineering. 127: 536-546.

[16] Sheikholeslami, M. \& Rokni, H. B. 2018. Numerical Simulation for Impact of Coulomb Force on Nanofluid Heat Transfer in a Porous Enclosure in Presence of Thermal Radiation. International Journal of Heat and Mass Transfer. 118: 823-831.

[17] You, S., Kim, J. \& Kim, K. 2003. Effect of Nanoparticles on Critical Heat Flux of Water in Pool Boiling Heat Transfer. Applied Physics Letters. 83: 3374-3376.

[18] Myers, J. R., P. D., Alam, T. E., Kamal, R., Goswami, D. \& Stefanakos, E. 2016. Nitrate Salts Doped with CuO Nanoparticles for Thermal Energy Storage with Improved Heat Transfer. Applied Energy. 165: 225-233.

[19] Sheikholeslami, M., Ghasemi, A., LI, Z., Shafee, A. \& Saleem, S. 2018. Influence of CuO Nanoparticles on Heat Transfer Behavior of PCM in Solidification Process Considering Radiative Source Term. International Journal of Heat and Mass Transfer. 126: 1252-1264.

[20] Akbari, O. A., Toghraie, D., Karimipour, A., Marzban, A. \& Ahmadi, G. R. 2017. The Effect of Velocity and Dimension of Solid Nanoparticles on Heat Transfer In Non-Newtonian Nanofluid. Physica E: Low-Dimensional Systems and Nanostructures. 86: 68-75.

[21] Minkowycz, W., Sparrow, E. M. \& Abraham, J. P. 2016. Nanoparticle Heat Transfer and Fluid Flow. CRC Press.

[22] Sheikholeslami, M., Hayat, T., Alsaedi, A. \& Abelman, S. 2017. Numerical Analysis of EHD Nanofluid Force Convective Heat Transfer Considering Electric Field Dependent Viscosity. International Journal of Heat and Mass Transfer. 108: 2558-2565.

[23] Esfe, M. H., Afrand, M., Gharehkhani, S., Rostamian, H., Toghraie, D. \& Dahari, M. 2016. An Experimental Study on Viscosity of Alumina-Engine Oil: Effects of Temperature and Nanoparticles Concentration. International Communications in Heat and Mass Transfer. 76: 202-208.

[24] Rasheed, A., Alias, H. \& Salman, S. 2018. Effects of Coil Pitch Spacing on Heat Transfer Performance of Nanofluid Turbulent Flow through Helical Microtube Heat Exchanger. International Journal of Engineering \& Technology. 7: 356360.

[25] Fule, P., Bhanvase, B. \& Sonawane, S. 2017. Experimental investigation of Heat Transfer Enhancement in Helical Coil Heat Exchangers Using Water based CuO Nanofluid. Advanced Powder Technology. 28: 2288-2294.

[26] Palanisamy, K. \& Kumar, P. 2017. Heat Transfer Enhancement and Pressure Drop Analysis of a Cone Helical Coiled Tube Heat Exchanger using MWCNT/water Nanofluid. Journal of Applied Fluid Mechanics. 10: 7-13.

[27] Bagherzadeh, F., Saffar-Avval, M., Seyfi, M. \& Abbassi, A. 2017. Numerical Investigation of Nanofluid Heat Transfer in Helically Coiled Tubes Using the Four-equation Model. Advanced Powder Technology. 28: 256-265.

[28] Reddy, V. K., Somanchi, N. S., Devi, S. R., Gugulothu, R. \& Kumar, S. P. 2015. Heat Transfer Enhancement in a Double Pipe Heat Exchanger Using Nanofluids. Proceedings of 17th ISME Conference on Advances in Mechanical Engineering. Department of Mechanical Engineering, Indian Institute of Technology, Delhi. 3-4.

[29] Salem, M., Ali, R., Sakr, R. \& Elshazly, K. 2015. Effect of $Y^{-}$ Al2O3/water Nanofluid on Heat Transfer and Pressure Drop Characteristics of Shell and Coil Heat Exchanger with Different Coil Curvatures. Journal of Thermal Science and Engineering Applications. 7: 041002.

[30] Sidik, C., Azwadi, N. \& Raad Abdulwahab, M. 2016. Numerical Investigation of Turbulent Magnetic Nanofluid 
Flow Inside Straight Channels. Applied Mechanics and Materials. Trans Tech Publ. 382-391.

[31] Arani, A. A. A., Aberoumand, H., Jafarimoghaddam, A. \& Aberoumand, S. 2017. Mixed Convection Heat Transfer: An Experimental Study on Cu/Heat Transfer Oil Nanofluids Inside Annular Tube. Heat and Mass Transfer. 53: 2875-2884

[32] Kumar, N. R., Bhramara, P., Addis, B. M., Sundar, L. S., Singh, M. K. \& Sousa, A. C. 2017. Heat Transfer, Friction Factor and Effectiveness Analysis of Fe3O4/water Nanofluid Flow in a Double Pipe Heat Exchanger with Return Bend. International Communications in Heat and Mass Transfer. 81: 155-163.

[33] Etghani, M. M. \& Baboli, S. A. H. 2017. Numerical Investigation and Optimization of Heat Transfer and Exergy Loss in Shell and Helical Tube Heat Exchanger. Applied Thermal Engineering. 121: 294-301.

[34] Wen, J., Huang, H., FU, Y., Xu, G. \& Zhu, K. 2017. Heat Transfer Performance of Aviation Kerosene RP-3 Flowing in a Vertical Helical Tube at Supercritical Pressure. Applied Thermal Engineering. 121: 853-862.

[35] Panahi, D. \& Zamzamian, K. 2017. Heat Transfer Enhancement of Shell-and-Coiled Tube Heat Exchanger Utilizing Helical Wire Turbulator. Applied Thermal Engineering. 115: 607-615.

[36] Zhang, C., Wang, D., Xiang, S., Han, Y. \& Peng, X. 2017. Numerical Investigation of Heat Transfer and Pressure Drop in Helically Coiled Tube with Spherical Corrugation. International Journal of Heat and Mass Transfer. 113: 332341.

[37] Fu, Y., Wen, J., Tao, Z., Xu, G. \& Huang, H. 2017. Surface Coking Deposition Influences on Flow and Heat Transfer of Supercritical Hydrocarbon Fuel in Helical Tubes. Experimental Thermal and Fluid Science. 85: 257-265.

[38] Palve, V. M. \& Kale, R. V. 2015. Computational Analysis of Helical Coil Heat Exchanger for Temperature and Pressure Drop. International Research Journal of Engineering and Technology. 1102

[39] Pilla, T. S., Sunkari, P. K. G., Padmanabhuni, S. L., Nair, S. S. \& Dondapati, R. S. 2017. Experimental Evaluation Mechanical Performance of the Compressor with Mixed Refrigerants R-290 and R-600a. Energy Procedia. 109: 113121.

[40] Šalić, A., Tušek, A. \& Zelić, B. 2012. Application of Microreactors in Medicine and Biomedicine. Journal of Applied Biomedicine. 10: 137-153.

[41] Dondapati, R. S., Saini, V., Kishore, N. \& Prasad, V. 2015 Enhancement of Performance Parameters of Transformer Using Nanofluids. Int J Sci Eng Technol. 4.

[42] Turkyilmazoglu, M. 2016. Performance of Direct Absorption Solar Collector with Nanofluid Mixture. Energy Conversion and Management. 114: 1-10.
[43] Dondapati, R. S., Saini, V., Verma, K. N. \& Usurumarti, P. R. 2017. Computational Prediction of Pressure Drop and Heat Transfer with Cryogen Based Nanofluids to be Used in Micro-Heat Exchangers. International Journal of Mechanical Sciences. 130: 133-142.

[44] Kandlikar, S. G., Colin, S., Peles, Y., Garimella, S., Pease, R. F., Brandner, J. J. \& Tuckerman, D. B. 2013. Heat Transfer in Microchannels-2012 Status and Research Needs. Journal of Heat Transfer. 135: 091001.

[45] Sivakumar, A., Alagumurthi, N. \& Senthilvelan, T. 2014. Experimental Investigation in Thermal Conductivity of Cuo and Ethylene Glycol Nanofluid in Serpentine Shaped Microchannel. International Journal of Engineering Science and Technology. 6: 430.

[46] Ebrahimi, A., Rikhtegar, F., Sabaghan, A. \& Roohi, E. 2016. Heat Transfer and Entropy Generation in a Microchannel with Longitudinal Vortex Generators Using Nanofluids. Energy. 101: 190-201.

[47] Akbari, O. A., Safaei, M. R., Goodarzi, M., Akbar, N. S. Zarringhalam, M., Shabani, G. A. S. \& Dahari, M. 2016. A Modified Two-phase Mixture Model of Nanofluid Flow and Heat Transfer in a 3-D Curved Microtube. Advanced Powder Technology. 27: 2175-2185.

[48] Behnampour, A., Akbari, O. A., Safaei, M. R., Ghavami, M. Marzban, A., Shabani, G. A. S. \& Mashayekhi, R. 2017. Analysis of Heat Transfer and Nanofluid Fluid Flow in Microchannels with Trapezoidal, Rectangular and Triangular Shaped Ribs. Physica E: Low-dimensional Systems and Nanostructures. 91: 15-31.

[49] Sajadifar, S. A., Karimipour, A. \& Toghraie, D. 2017. Fluid Flow and Heat Transfer of Non-Newtonian Nanofluid in a Microtube Considering Slip Velocity and Temperature Jump Boundary Conditions. European Journal of Mechanics-B/Fluids. 61: 25-32.

[50] Karimzadehkhovei, M., Sadaghiani, A. K., Motezakker, A. R., Akgönül, S., Ozbey, A., Sendur, K., Mengüç, M. P. \& Koşar, A. 2018. Experimental and Numerical Investigation of Inlet Temperature Effect on Convective Heat Transfer of Y-Al2O3/Water Nanofluid Flows in Microtubes. Heat Transfer Engineering. 1-15.

[51] Rahimi Gheynani, A., Ali Akbari, O., Zarringhalam, M. Ahmadi Sheikh Shabani, G., Alnaqi, A. A., Goodarzi, M. \& TOGHRAIE, D. 2018. Investigating the Effect of Nanoparticles Diameter on Turbulent Flow and Heat Transfer Properties of Non-Newtonian Carboxymethyl Cellulose/CuO fluid in a Microtube. International Journal of Numerical Methods for Heat \& Fluid Flow.

[52] Chamkha, A. J., Molana, M., Rahnama, A., Ghadami, F. 2018. On the Nanofluids Applications In Microchannels: A Comprehensive Review. Powder Technology. 332: 287-322. 